\title{
In vivo study on murine species using Cytarabine magnetic microspheres
}

\author{
Satinder Kakar', Anurekha Jain², Ramandeep Singh ${ }^{3}$ \\ 1,2 Jayoti Vidyapeeth Women University, India \\ ${ }^{3}$ Himachal Institute of Pharmacy, Paonta Sahib, India
}

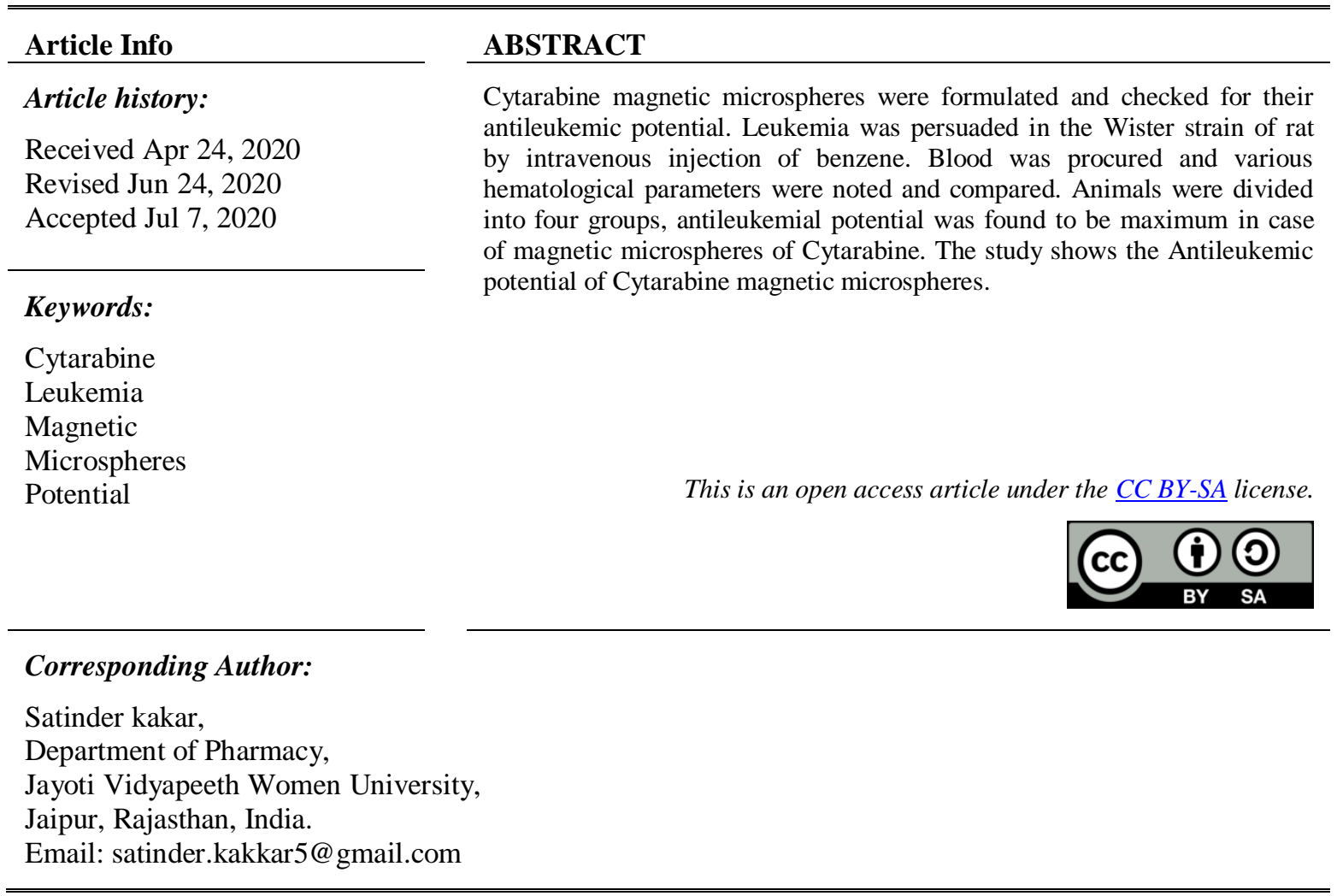

\section{INTRODUCTION}

Magnetic microspheres are the particles that are miniature to circulate through the capillaries but are sufficiently receptible to be encaptured in microvessels by applying magnetic fields of $0.4 \mathrm{~T}-0.8 \mathrm{~T}$ [1]. Success has achieved in dealing with diseases by invention of drugs in histories, which are fulfilling the summons of present day drug therapy i.e. modification of the drug action along with lessening of toxic side effects in vivo. Recently a lot of interest has been shown in targeted drug delivery system magnetic microspheres are the one. Targeting i.e. integration of magnetic particles into polymers is covered in MMs. Need for magnetic microspheres: 1) orally administrable pharmaceutical compositions ineffective as a result of absorption of pharmacological active agent in the digestive tract before the target site are reached. 2) Rectal delivery-less convenient and less acceptable than oral formulations. 3) Colon specific drug delivery system-do not delivers the right amount to colon due to RES Clearance. 4) Non magnetic microspheres-cleared off by the RES system. 5) In case of liposomes, resealed erythrocytes the drug carrier suffer major stability problem, hence shelf life is reduced, and so special storage conditions are needed which is not viable. 6) While in Monoclonal Antibodies preparation, selection and isolation of an appropriate antigen for developing monoclonal antibody is again a brain taxing problem, show bad site specificity and are quickly cleared off by Reticuloendothelial system.

However magnetic microspheres show good site specificity and RES Clearance is also minimized. In order to prove any work animal activity is performed thus in vivo study of Cytrabine has been performed. Various data reported have shown that benzene is a slow acting poison. It affects the bone marrow part a lot. 
The peril of benzene is joined with cumulative exposures of benzene [2]. As Cytarabine is a proved drug for leukemia, therefore it was induced to treat leukemia induced in mice [3-5]. Antileukemic activity of Cytarabine magnetic microsphere is the basic aim for the study.

\section{RESEARCH METHOD}

Cytarabine was procured from Avanscure Lifesciences Pvt Ltd and magnetic microspheres were formulated for it using continuous solvent evaporation method. In order to check the activity of these prepared formulations in vivo study was performed on wistar rats. Wistar strain of rats was used for the study. The protocol for performing experiment was approved by Institutional Animal Ethical Committee as per the rules of CPCSEA, India.

Animals were euthanized after accomplishment of the study and the carcasses were disposed as per the guidelines of the institute. Leukemia was persuaded in Wister rats by intravenous administration of $0.2 \mathrm{ml}$ of a 1:10 diluted benzene solution (chloroform in water/2-propanol [50/50] v/v), given each 2 days for 3 weeks successively [5]. Cytarabine magnetic microspheres were administered prior to, during, and ensuing leukemia creation. Leukemia burden was noted by comparing the hematological parameters at baseline and after leukemia induction in various experimental groups. Succeding 3 weeks of benzene injection, rats in the different groups were bled by cardiac puncture. The blood was collected into EDTA vials, mixed, labeled, and checked [6, 7]. Samples were analyzed for packed cell volume, WBCs, RBCs, hemoglobin, platelets, lymphocytes, RBC distribution width, and mean platelet volume [8-10].

\section{RESULTS AND DISCUSSION}

The results obtained from the study are summarized in Table 1.Significant increase in the parameters such as RBCs, hemoglobin, WBCs and platelets was seen on administration of Cytarabine magnetic microspheres, as the target site specificity is enhanced with the magnetic formulations. Also Reticuloendothelial clearance can be minimized. Thus these formulations show leukemogenic effects.

Table 1. Hematological parameters at baseline, postleukemia induction, and after treatment with treatment

\begin{tabular}{cccccc}
\multicolumn{5}{c}{ with Cytarabine magnetic microspheres } \\
\hline Groups & Sample & Red blood cells & Hemoglobin & White blood cells & Platelets \\
\hline \multirow{2}{*}{ Normal Control } & Baseline & $8.68 \pm 0.93$ & $15.61 \pm 0.65$ & $10.52 \pm 0.49$ & $268.37 \pm 18.25$ \\
& Postanalytical & $8.91 \pm 0.06$ & $15.82 \pm 0.55$ & $10.95 \pm 0.06$ & $291.30 \pm 18.60$ \\
Leukemia Control & Baseline & $8.29 \pm 0.14$ & $15.40 \pm 0.86$ & $11.77 \pm 0.30$ & $241.96 \pm 18.05$ \\
Cytarabine magnetic & Postanalytical & $5.18 \pm 0.02$ & $12.65 \pm 0.18^{*}$ & $17.04 \pm 0.11$ & $254.49 \pm 18.55$ \\
microspheres (50 mg/kg) & Baseline & $9.04 \pm 0.51$ & $14.88 \pm 0.47$ & $10.05 \pm 0.82$ & $295.29 \pm 19.66$ \\
& Postanalytical & $8.87 \pm 0.61^{* * *}$ & $14.58 \pm 0.37$ & $9.38 \pm 0.31^{*}$ & $386.47 \pm 26.71 * *$ \\
Cytarabine & Baseline & $8.38 \pm 0.29$ & $14.78 \pm 0.01$ & $10.06 \pm 0.19$ & $273.41 \pm 19.43$ \\
& Postanalytical & $7.01 \pm 0.82^{*}$ & $13.67 \pm 0.97^{* *}$ & $10.56 \pm 0.74 * *$ & $251.37 \pm 18.20$ \\
\hline
\end{tabular}

Results are given as mean $\pm \operatorname{SEM}(n=6)$. Normal control group contrasted with rest of the treated groups with the significance at $* P<0.05, * * P<0.01, * * * P<0.001$. In time to come by merging different additional policies, microspheres will have chief position in new drug delivery, especially in diseased cell finding, targeted and effective in vivo delivery and promoters as mini models of diseased organ and tissues [11-13]. Magnetic Microsphere gives huge chances for formulating new controlled and delayed release formulations, thus extending the borderline of upcoming pharmaceuticals [14-16]. This dosage form provides numerous advantages such as shielding and masking, reduced dissolution rate, easing and handling; spatial targeting of the drug. This system helps in precise delivery of less quantities of potent drugs, diminished drug concentrations at sites other than the target tissue; and protection of unbalanced compounds before and after administration and prior to appearance at the site of action. A research was presented by Gerald G Enriquez, 2013 capable of efficiently delivering sulforaphane, a histone deacetylase inhibitor, for an extended period of time in vivo, similarly effect can be obtained for leukemia thus Cytarabine magnetic microspheres are formulated [17]. Magnetic Microsphere technique will discover the important place in new drug delivery system. Especially these can be used for bed ridden patients [18]. Solely, the magnetic microsphere carrier will be broadly utilized in the biomedical field as a beneficial drug carrier [19, 20]. Contrasting with traditional systems; biomass-based drug delivery systems shows higher-ranking drug safety due to their very little pharmacological effects with the matrix [21-24]. Magnetism seems to be a common function of opening a new vista of a multi-barrier of multi-step drug delivery. Their benefit is the 
targeting of drug using an external magnet, which can be done very easily. They are relatively novel drug delivery systems, having received attention from the early 1990s. In the early days of twentieth century, Paul Ehrlich envisioned his magic bullet concept-the idea that drugs reach the right site in the body, at the right time, at right concentration [25].

\section{CONCLUSION}

Benzene is a solvent that is shown to cause leukemia as reported in vast literature studies. Leukemia most commonly it affects the white blood cells. If a person is having problem with the production of RBCs then he may carry leukemia as a disease in future, although there are oral dosage forms, non magnetic microspheres but they are ineffective in targeting the drug to the target site; also they are cleared by the Reticuloendothelial clearance. Thus site specificity is decreased; in order to overcome the magnetic forms were prepared to increase the target site specificity and to minimize RES clearance. Thus there are many future prospects for magnetic microspheres as cancer is the leading disease now a days, it has increased the mortality rate.

Cytarabine is poorly absorbed from the GIT; it shows very good solubility in water. The half life elimination varies in patients depending on the age and it varies from 1-3 hours. As the bioavailability of orally administered Cytarabine is very low thus novel delivery system that is magnetic microspheres have been formulated. In each delivery system therapeutic amount of drug is needed at the needed site. Targeting is drug delivery to the chosen body part to which one wants to deliver the drug. The wanted drug distribution by targeted delivery would leave the rest of the body and thus lowers the overall toxicity while maintaining its therapeutic benefits. Many clinical schemes need delivery of agents that are therapeutic at the desired delivery point. An attempt was made to prepare Cytarabine magnetic microspheres in order to investigate targeting efficiency, enhance bioavailability, and reduce dose and thereby improving patient compliance. Targeting by means of magnetic fields seems to be a vital and most common function of opening a new vista of a multi-barrier of multi-step drug delivery. Their main advantage is the targeting of drug using an external magnet, which can be accomplished very easily thus Reticuloendothelial clearance can be minimized and target site specificity can be increased. Magnetic microspheres are novel drug delivery systems, having received attention from the early 1990s. Thus magnetic microspheres have the great potential for these objectives. This is an effective tool for the cancer patients. The future holds agreement in magnetic microspheres and by supplemental study this will be developed as novel and efficient approach for targeted drug delivery system.

\section{REFERENCES}

[1] Satinder, Kakar, Ramandeep, Singh., " Preparation of magnetic microspheres of mesalamine by phase separation emulsion polymerization technique," African Journal of Pharmacy and Pharmacology, vol. 8, no. 9, pp. 246-258, 2014.

[2] Ling-li ,Ye., et al., "Are polymorphisms in metabolism protective or a risk for reduced white blood cell counts in a Chinese population with low occupational benzene exposures?," International Journal of Occupational and Environmental Health, vol. 21, no. 3, pp. 232-240, 2015.

[3] Satinder, Kakar, Anurekha, Jain, "Magnetic microspheres: an Overview," Asian Pac. J. Health Sci., vol. 6, no. 1, pp. 81-89, 2019.

[4] Morgane, Gourvest, Pierre, Brousset., Marina, Bousquet., "Long Noncoding RNAs in Acute Myeloid Leukemia: Functional Characterization and Clinical Relevance," Cancers, vol. 11, no. 11, pp. 1638, 2019.

[5] Julie Tzu-Wen, et al., "Engineering red-emitting multi-functional nano capsules for magnetic tumour targeting and imaging," Biomaterials Science, vol. 8, no. 9, pp. 2590-2599, 2020.

[6] Olufemi, AE., Terry, AO., Kola, OJ., "Anti-leukemic and immune modulatory effects of fungal metabolites of Pleurotuspulmonarius and Pleurotusostreatuson benzene-induced leukemia in Wister rats," Korean J Hematol., vol. 47, no. 1, pp. 67-73, 2012.

[7] Smith, MT., "The mechanism of benzene-induced leukemia: A hypothesis and speculations on the causes of leukemia," Environ Health Perspect, vol. 104, Suppl 6, pp. 1219-1225, 1996.

[8] Mahnaz, Amiri, Masound, Salavati, Niasari, Ahmad, Akbari, "Magnetic nanocarriers: Evolution of spinel ferrites for medical applications," Advances in Colloid and Interface Science, vol. 265, pp. 29-44, 2019.

[9] Nattawut Leelakanok, Sean Geary, Aliasger Salem, "Fabrication and Use of Poly (D,L-lactide-co-glycolide)-Based Formulations Designed for Modified Release of 5-Fluorouracil," Journal of Pharmaceutical Sciences, vol. 107, no. 2, pp. 513-528, 2018.

[10] Ge, J., et al., "Initiation of targeted nano drug delivery in vivo by a multifunctional magnetic implant," ACS Appl. Mater. Interfaces, vol. 9, no. 24, pp. 20771-20778, 2017. 
[11] Lengyel, M., et al."'Microparticles, Microspheres, and Microcapsules for Advanced Drug Delivery,” Sci. Pharm., vol. 87, no. 3, pp. 20, 2019.

[12] Farah, F.H., "Magnetic Microspheres: A Novel Drug Delivery System," World, J. Pharm. Pharm. Sci. vol. 3, pp. 93-112, 2017.

[13] Bouman, J., "Controlled Release from Zein Matrices: Interplay of Drug Hydrophobicity and pH," Pharm. Res. vol. 33, no. 3, pp. 673-685, 2016.

[14] Upadhyay, A., et al., "Effect of PEGylation on performance of protein microbubbles and its comparison with lipid microbubbles," Mater. Sci. Eng. C Mater Biol Appl, vol. 71, pp. 425-430, 2017.

[15] Wu, D., Duan, C., Chen, L. et al. "Efficacy and safety of different doses of cytarabine in consolidation therapy for adult acute myeloid leukemia patients: a network meta-analysis," Sci. Rep., vol. 7, no. 1, pp. 9509, 2017.

[16] Papaemmanuil E., et al., "Genomic classification and prognosis in acute myeloid leukemia," $N$ Engl J Med., vol. 374, no. 23, pp. 2209-2221, 2016.

[17] Gerald G Enriquez, et al., "Formulation and evaluation of drug-loaded targeted magnetic microspheres for cancer therapy," Int J Nanomedicine, vol. 8, pp. 1393-1402, 2013.

[18] Hyma, P., et al.; "Formulation and evaluation of sodium alginate based Ramipril buccal films," Journal of Chemical and Pharmaceutical Research, vol. 8, no. 5, pp. 765-770, 2016.

[19] Hee Man Kim., "Raft Formation of Sodium Alginate in the Stomach," J Neurogastroenterol Motil, vol. 22, no. 4, pp. 705-706, 2016.

[20] Xin, Li., et al., "Synthesis and characterization of magnetic chitosan microspheres for drug delivery," RSC Advances, vol. 10, no. 12, pp. 7163, 2020.

[21] Rastogi, V., Shukla, S., Singh,R., "Microspheres: A Promising Drug Carrier," Journal of Drug Delivery and Therapeutics, vol. 6, no. 3, pp. 18-26, 2016.

[22] Yadav, R., Bhowmick ,M., Rathi, Rathi, J., "Design and characterization of floating microspheres for rheumatoid arthritis," Journal of Drug Delivery and Therapeutics, vol. 9, no. 2-s, pp. 76-81, 2019.

[23] Nagasree K., Chowdary G. V., Mahendra Kumar, C. B., "Design and evaluation of sodium alginate microspheres loaded with gatifloxacin," Der Pharmacia Lettre, vol. 8, no. 4, pp. 361-370, 2016.

[24] Mikhaylov,G., Mikac,U., Magaeva, "Ferri-liposomes as an MRI-visible drug-delivery system for targeting tumours ISRN Nanomaterials and their microenvironment," Nature Nanotechnology, vol. 6, no. 9, pp. 594-602, 2011.

[25] Ayyanaar, S., et al., "Iron oxide nanoparticle core-shell magnetic microspheres: Applications toward targeted drug delivery, Nanomedicine,” Nanotechnology, Biology, and Medicine, vol. 24, pp. 102134, 2019. 